\title{
Detection of Nitroaromatic and Peroxide Explosives in Air Using Infrared Spectroscopy: QCL and FTIR
}

\author{
Leonardo C. Pacheco-Londoño, John R. Castro-Suarez, and Samuel P. Hernández-Rivera
}

Department of Chemistry, ALERT-DHS Center of Excellence, Center for Chemical Sensors Development, University of Puerto Rico at Mayagüez, P.O. Box 9000, Mayagüez, PR 00681-9000, USA

Correspondence should be addressed to Samuel P. Hernández-Rivera; samuel.hernandez3@upr.edu

Received 4 October 2012; Revised 26 February 2013; Accepted 11 March 2013

Academic Editor: Augusto Belendez

Copyright ( 2013 Leonardo C. Pacheco-Londoño et al. This is an open access article distributed under the Creative Commons Attribution License, which permits unrestricted use, distribution, and reproduction in any medium, provided the original work is properly cited.

\begin{abstract}
A methodology for processing spectroscopic information using a chemometrics-based analysis was designed and implemented in the detection of highly energetic materials (HEMs) in the gas phase at trace levels. The presence of the nitroaromatic HEM 2,4-dinitrotoluene (2,4-DNT) and the cyclic organic peroxide triacetone triperoxide (TATP) in air was detected by chemometricsenhanced vibrational spectroscopy. Several infrared experimental setups were tested using traditional heated sources (globar), modulated and nonmodulated FT-IR, and quantum cascade laser- (QCL-) based dispersive IR spectroscopy. The data obtained from the gas phase absorption experiments in the midinfrared (MIR) region were used for building the chemometrics models. Partial least-squares discriminant analysis (PLS-DA) was used to generate pattern recognition schemes for trace amounts of explosives in air. The QCL-based methodology exhibited a better capacity of discrimination for the detected presence of HEM in air compared to other methodologies.
\end{abstract}

\section{Introduction}

The detection of highly energetic materials (HEMs) at trace levels in air remains a subject of great importance to national defense and security. In the past few years, most of the published reports have focused on the detection of these important chemical compounds. However, the majority of them require some type of sampling $[1,2]$. Obtaining samples in the field is the principal disadvantage of most explosive detection devices because the person doing the sampling is at risk.

Most of the analytical techniques employed for the development of methodologies for HEM detection are based on spectroscopic and chromatographic techniques $[1,2]$. Trace amounts of 2,4-dinitrotoluene (2,4-DNT) in air have been detected and discriminated by surface-enhanced Raman spectroscopy (SERS) using a gold surface sensor [3]. These sensors generate a response in the presence or absence of 2,4-DNT and other volatile nitroaromatic HEMs in air. In this case, the sample vapor was introduced to the sensor with a fan. High-speed fluorescence spectroscopy is another method for the detection of nitroaromatic HEM in the air. This method employs silica microspheres coated with a highly sensitive fluorescent polymer that responds by quenching the fluorescence when HEM molecules attach to the polymer $[1,2,4-7] .2,4$-DNT can also be detected and quantified by measuring the IR acoustic wave in polymercoated surfaces [8]. In this method, the presence of 2,4-DNT generates a change in the frequency of the acoustic wave on the surface, and this change is used for detection and quantification.

Air sampling with a solid-phase extraction cartridge to collect a toluene/methyl-tert-butyl ether analyte using a modified supercritical fluid extraction apparatus followed by separation and measurement of the extracted analyte by GC has also been used for the analysis of nitroaromatic HEM [9]. The detection of nitroaromatic HEM in air can also be performed by extraction with C-18 solid-phase membranes. In this case, the analyte is desorbed directly in a chromatographic mobile phase [10]. The detection of triacetone triperoxide (TATP) 
in air has been reported using a gas-washing sampling technique [11]. HPLC with postcolumn UV irradiation and photometric detection following photochemical degradation of TATP has also been used for detection [11, 12].

The use of chemometrics (multivariate analysis) with spectroscopic data in HEM detection has been very valuable because it has allowed for the exploration of very complex ambient matrices [13, 14]. Many chemometrics tools have been developed and tested. However, the most commonly used tools employed to identify, quantify, and classify datasets are those that make use of principal components analysis (PCA), partial least-squares (PLS), and discriminant analysis (DA), as well as their combined usage in PLS-DA and hierarchical cluster analysis (HCA). PCA transforms a set of variables into fewer variables (called dimensions, principal components, or components) that contain most of the information (variance) from the initial dataset. The PCA algorithm seeks to save the information from a large number of variables in a small number of uncorrelated components with minimal loss of information. One of the main reasons for performing a PCA is to reduce the number of variables to a few uncorrelated dimensions that contain as much information as possible (used to avoid multicollinearity in multiple regressions, among other things) [14]. PLS is a linear analysis routine that is used to design and build robust calibration models for quantitative analysis. PLS regression analysis is a quantitative spectral decomposition technique that is closely related to PCA regression [15]. PLS uses the concentration information during the decomposition process, which causes the spectra containing higher constituent concentrations to be weighted more heavily than those with lower concentrations. The main idea of PLS is to obtain as much information about the concentration as possible into the first few latent variables (number of components) [16]. Linear discriminant analysis (LDA or DA) is a multivariate technique that allows for the differentiation of separate objects from distinct populations and allocates new objects into populations previously defined [17]. The usefulness of this methodology is to determine a relationship of belonging or not belonging to a previously defined group.

The application of pattern recognition to infrared spectroscopy can be found in the current literature. PCA was used to analyze the FTIR spectra of mixtures of two monomers [18]. Discrimination between mayonnaise samples that contained different vegetable oils was achieved by PLS-DA of near infrared spectral data [19]. PLS-LDA has also been used in other areas of science and engineering, including biomedical studies such as the classification of tumors [20], early detection of diabetes related to changes in the skin [21], and fault diagnosis in chemical processes [22].

Detection and discrimination of HEM are important in the applications for national defense and security. Being able to detect and prevent a chemical/biological threat long before any damage to civilians, military personnel, and private or public property is a goal of agencies in charge of public security and national defense. To a large extent, remote detection modalities will benefit from chemometricsenhanced spectroscopic detection.
In this paper, infrared vibrational detection of highly energetic materials, such as 2,4-DNT and TATP, present in the gas phase in air was performed. The detection experiments were performed in the active mode using two source types including a traditional globar IR source for detection using FT-IR spectrometers (for both modulated and unmodulated lights) and a quantum cascade laser (QCL) IR source for detection with a dispersive spectrometer. PLSDA was used to generate pattern recognition schemes for trace amounts of explosives in air from the obtained IR spectra. Classificatory capacities from different models based on PLS-DA were used to establish the best experimental setup for the detection and classification of these explosives in the gas phase.

\section{Experimental Section}

2.1. Materials. The reagents used in this investigation included acetone $\left(\mathrm{CH}_{3} \mathrm{COCH}_{3}, 98 \% \mathrm{w} / \mathrm{w}\right.$, Sigma-Aldrich Chemical Co., Milwaukee, WI, USA), hydrogen peroxide $\left(\mathrm{H}_{2} \mathrm{O}_{2}, 50 \%\right.$ in water, Sigma-Aldrich), hydrochloric acid (HCl, $12 \mathrm{M}$, Merck, VWR, Inc., West Chester, PA, USA), sulfuric acid $\left(\mathrm{H}_{2} \mathrm{SO}_{4}, 18 \mathrm{M}\right.$, Merck), and dimethyl ether $\left(\mathrm{CH}_{3} \mathrm{OCH}_{3}\right.$, Sigma-Aldrich). Standard solutions of 2,4-DNT 1000 parts per million (ppm) GC/MS primary standards were obtained from Restek Corp. (Bellefonte, PA, USA) and from Chem Service, Inc. (West Chester, PA, USA). Crystalline samples of 2,4-DNT were purchased from Chem Service, Inc. (West Chester, PA, USA).

2.2. Synthesis of TATP. TATP cannot be purchased from chemical reagents suppliers. Therefore, it was prepared in small quantities as needed due to the high thermal instability of this powerful explosive. A white crystalline precipitate was obtained using a conventional synthesis method. The crystals were filtered and washed using distilled water followed by recrystallization from methyl ether.

2.3. Instrumentation. The IR equipment used for the experiments was a Bruker Optics (Billerica, MA, USA) model IFS 66v/S Fourier transform IR (FT-IR) interferometer. This system has an evacuable bench equipped with several sources, detectors, beam splitters, and other accessories to perform experiments on solid, liquid, and gas samples from the far IR region (to $50 \mathrm{~cm}^{-1}$ ) to the near IR region (as high as $7500 \mathrm{~cm}^{-1}$ ). For the experiments described, the system was equipped with a deuterated triglycine sulfate (DTGS) detector and a potassium bromide (KBr) beam splitter. An EM-27 open path (OP) FT-IR interferometer (Bruker Optics) was used to obtain the IR spectral information from the TATP samples with a thermoelectrically (TE) cooled mercurycadmium telluride (MCT) detector.

A LaserTune IR dispersive spectrometer equipped with a TE-cooled MCT detector (Block Engineering, Marlborough, MA, USA) was employed to obtain spectral information of the TATP samples. An Agilent 6890 gas chromatograph (GC) coupled to an Agilent 5893 mass selective detector (MSD) was used for qualitative analyses. An Agilent Technologies model 


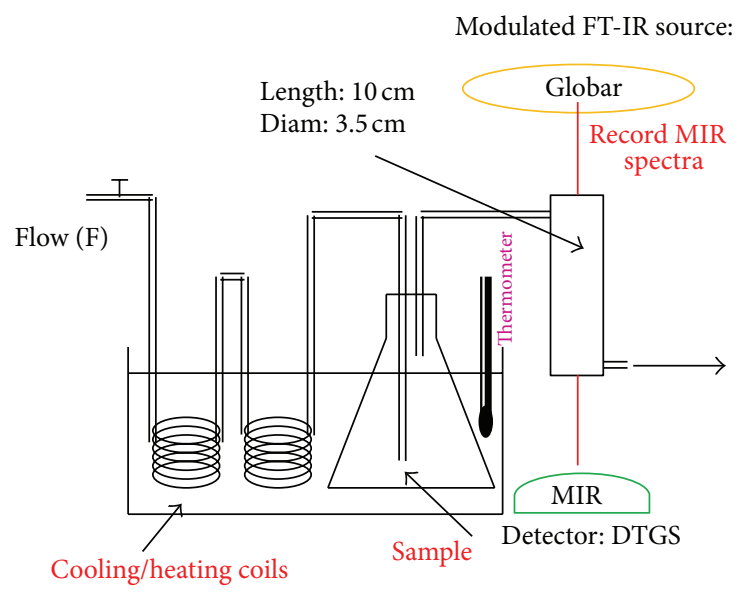

(a)

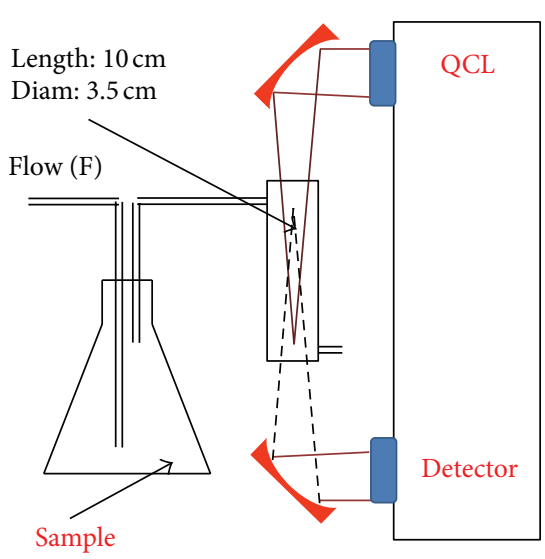

(c)

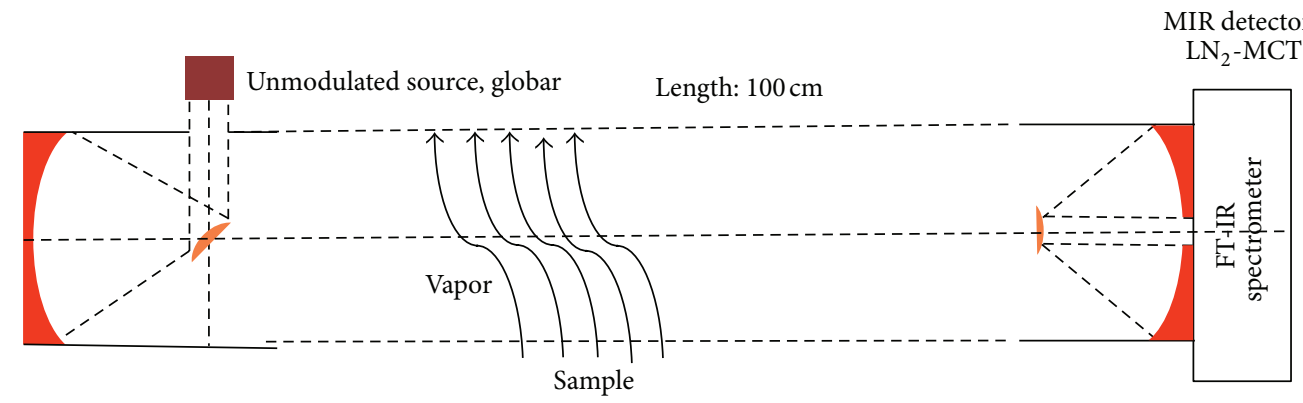

(b)

FIGURE 1: Schematic diagram of the experimental setup. (a) FT-IR interferometer with modulated light source. (b) Open Path FT-IR with unmodulated (external) thermal source. (c) Quantum cascade laser (QCL) scanning system.

$6890 \mathrm{~N}$, Network GC system with microcell ${ }^{63} \mathrm{Ni}$ Electron Capture Detector $(\mu$-ECD), was also used for the quantitative analyses. A capillary column HP-5 MS 5\% phenyl methyl siloxane, $30 \mathrm{~m}, 250 \mu \mathrm{m}$ in diameter, and $0.25 \mu \mathrm{m}$ of film thickness, was used.

\section{Measurements and Analysis}

3.1. Experiment. Figure 1 shows the schematic diagram of the experimental setup used in the investigation. Three types of experiments were performed. The setup for the first experiment is shown in Figure 1(a). The HEM samples were placed on the bottom of $500 \mathrm{~mL}$ Erlenmeyer flasks. A flow of dry air $(1-16 \mathrm{~mL} / \mathrm{s})$ at several temperatures $\left(0-38^{\circ} \mathrm{C}\right)$ was used. The temperature was regulated by either scanning in the range of temperature or by using point-by-point fixed temperature measurements. Trace amounts of explosives in the gas phase were dragged from the surface by the air flow and transported to an IR gas cell for detection. Spectra were recorded using the instrument at $4 \mathrm{~cm}^{-1}$ of resolution and 25 scans. The spectral range was $400-4000 \mathrm{~cm}^{-1}$.

The number of spectra obtained was 799 for 2,4-DNT/air; 120 for TATP/air; and 1881 spectra of ambient air. Figure 1(b) shows a schematic representation of the EM-27 interferometer setup employed to collect absorption spectra excited by a globar source. All of the active mode experiments were performed at ambient temperature $\left(25^{\circ} \mathrm{C}\right)$ at 30 scans and $4 \mathrm{~cm}^{-1}$ resolution. Sets of 100 air spectra, air with TATP, and air with DNT with a spectral range of $650-4000 \mathrm{~cm}^{-1}$ were collected.

A third experiment was performed using a quantum cascade laser (QCL) as the source with the Block Engineering LaserTune spectrometer. All of the active mode experiments were performed at the laboratory temperature of $20^{\circ} \mathrm{C}$ with $1 \mathrm{scan}$ at $4 \mathrm{~cm}^{-1}$ resolution. The spectral range was from 830 to $1430 \mathrm{~cm}^{-1}$. Forty-four spectra of air with 2,4-DNT, 25 spectra of air with TATP, and 37 spectra of air were obtained. The presence of TATP in the air was determined by GC-MS, and the concentrations of 2,4-DNT in the air for different fluid conditions were calculated using a calibration curve from GC- $\mu \mathrm{ECD}$, which was only performed for the first experiment.

3.2. Partial Least-Squares (PLS) Discriminant Analysis (DA). PLS-DA runs were performed using the OPUS 6.0 software (Bruker Optics) combined with the Statgraphics Plus v. 15.2 (StatPoint Technologies, Inc., Warrenton, VA, USA) statistical analysis software. The models were evaluated using internal validation, statistical significance $(P)$, and the percentage of cases correctly classified (PCCC). For internal 

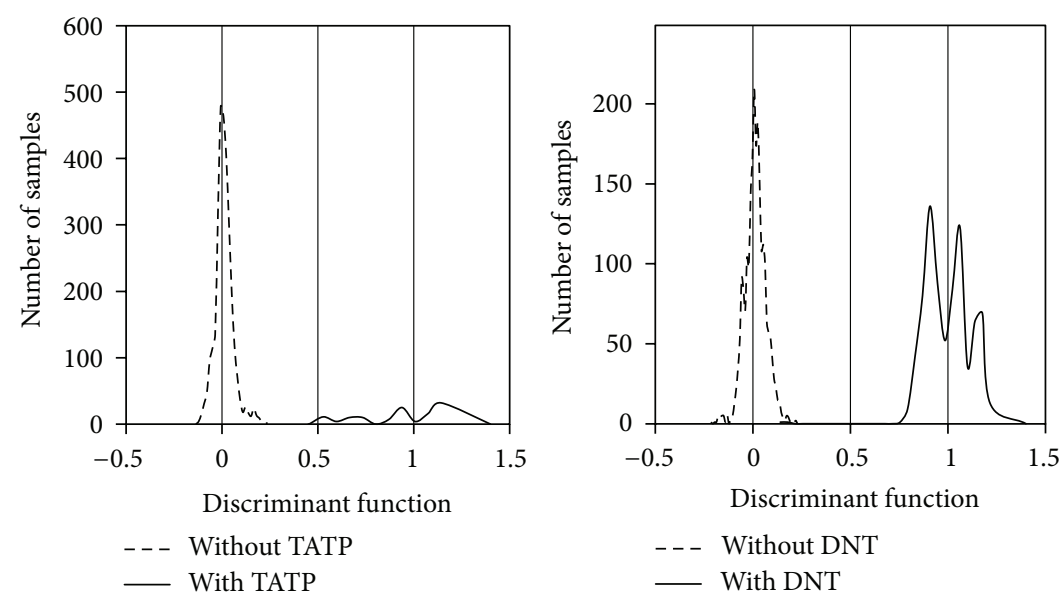

(a)
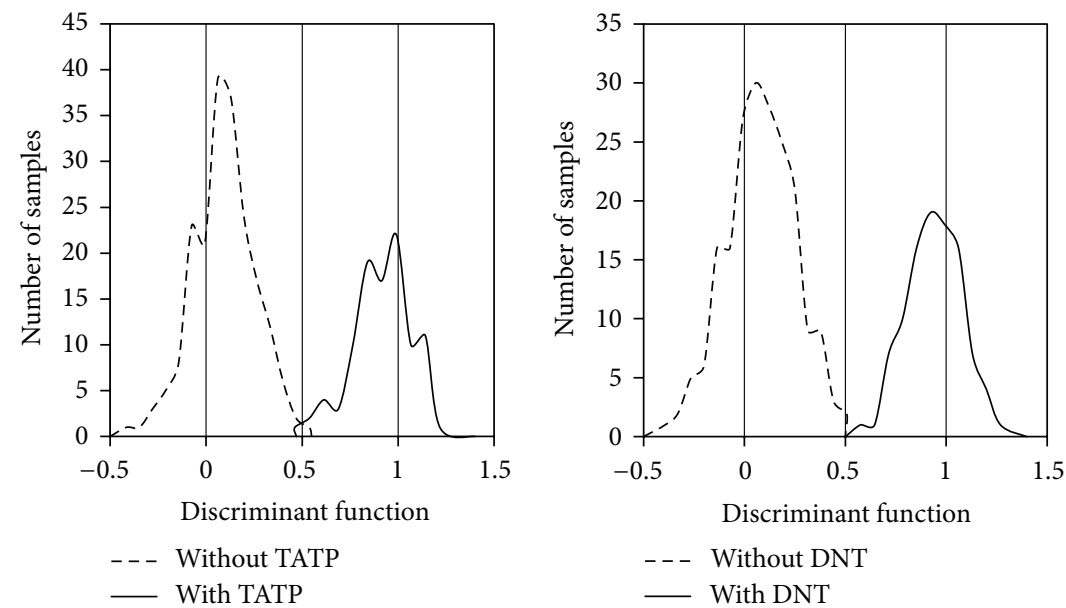

(b)
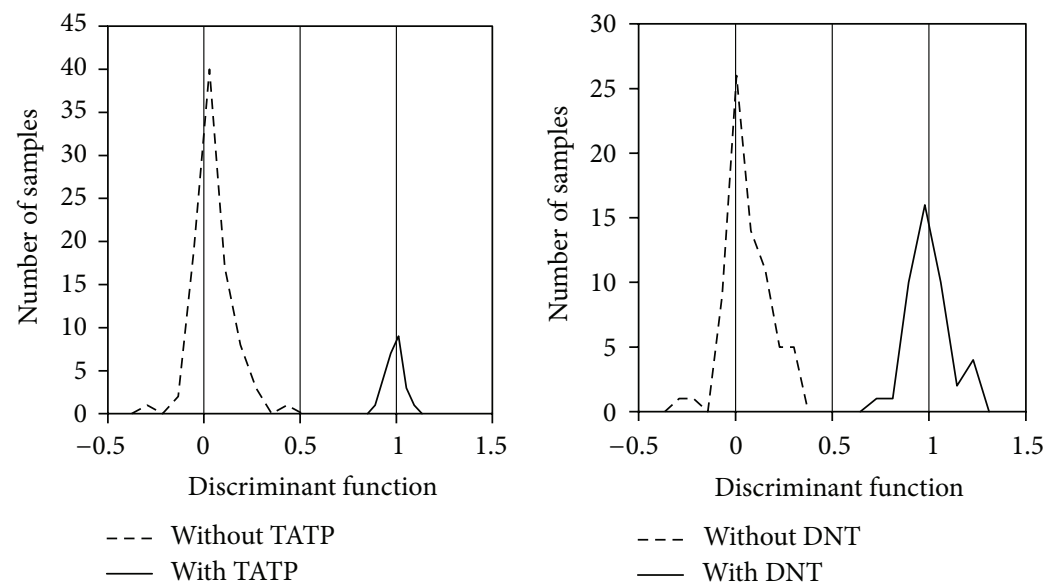

(c)

Figure 2: (a) Histogram for discrimination using modulated source FT-IR. (b) Histogram for discrimination using unmodulated thermal source FT-IR. (c) Histogram for discrimination using QCL. 
TABLE 1: Validation parameters for the various models constructed.

\begin{tabular}{|c|c|c|c|c|c|c|}
\hline \multirow{2}{*}{ Parameter } & \multicolumn{2}{|c|}{ Modulated FTIR (model 1) } & \multicolumn{2}{|c|}{ Unmodulated FTIR (model 2) } & \multicolumn{2}{|c|}{ QCL scan (model 3) } \\
\hline & TATP & DNT & TATP & DNT & TATP & DNT \\
\hline Wilks' lambda & 0.12 & 0.03 & 0.09 & 0.11 & 0.05 & 0.06 \\
\hline Canonical correlation & 0.94 & 0.98 & 0.96 & 0.94 & 0.98 & 0.97 \\
\hline PCCC & $100.00 \%$ & $100.00 \%$ & $100.00 \%$ & $100.00 \%$ & $100.00 \%$ & $100.00 \%$ \\
\hline PCCCC & $100.00 \%$ & $99.94 \%$ & $100.00 \%$ & $99.97 \%$ & $100.00 \%$ & $100.00 \%$ \\
\hline Sensibility & $100.00 \%$ & $100.00 \%$ & $100.00 \%$ & $99.50 \%$ & $100.00 \%$ & $100.00 \%$ \\
\hline Specificity & $100.00 \%$ & $99.75 \%$ & $100.00 \%$ & $100.00 \%$ & $100.00 \%$ & $100.00 \%$ \\
\hline False alarm & $0.00 \%$ & $0.00 \%$ & $0.00 \%$ & $0.50 \%$ & $0.00 \%$ & $0.00 \%$ \\
\hline Missed detection & $0.00 \%$ & $0.25 \%$ & $0.00 \%$ & $0.00 \%$ & $0.00 \%$ & $0.00 \%$ \\
\hline Loadings & 7 & 7 & 10 & 10 & 5 & 5 \\
\hline Samples $(N)$ & 3079 & 3079 & 300 & 300 & 106 & 106 \\
\hline$P$ value & $<0.0001$ & $<0.0001$ & $<0.0001$ & $<0.0001$ & $<0.0001$ & $<0.0001$ \\
\hline
\end{tabular}

validation, each spectrum was successively removed from the dataset and discriminated from a new model built from the remaining spectra. This procedure was performed for each of the spectra in the dataset, and the predicted discrimination was compared with the experimental observations. The generated percentage of cases correctly classified is called the cross-percentage of cases correctly classified (PCCCC) or the leave-one-out cross-validation (LOOCV or CV) procedure. The other statistical indicators that were used included the Wilks' lambda and canonical correlation.

\section{Results and Discussion}

Figure 2 shows the frequency distribution for the CV of air with TATP and DNT. The solid line represents the data for air with the analyte of interest, and the dotted line represents the data for clean air and air with other analytes. Good discrimination was obtained in all of the statistical experiments. The evaluation is shown in Table 1. The PCCC for all of the models was $100.0 \%$, and complete classification is observed. However, the cross-validation PCCCC was not $100.0 \%$ for DNT samples.

In the FT-IR model with a modulated globar source, the PCCCC for TATP was $100 \%$ but it was lower for 2,4-DNT. This result can be attributed to the fact that pure air was only analyzed at $25^{\circ} \mathrm{C}$, whereas 2,4-DNT was analyzed from $0^{\circ} \mathrm{C}$ to $38^{\circ} \mathrm{C}$. In this model for $2,4-\mathrm{DNT}, 0.25 \%$ of the sample was not correctly classified. These data missed the detection of or indicated a false negative for air with 2,4-DNT at low temperatures where the sublimation of DNT is very small. For this model of 2,4-DNT, the sensitivity was $100.00 \%$, the specificity was $99.75 \%$, and the false alarm rate was $0.00 \%$.

In the second model (unmodulated FT-IR), the PCCCs for all of the models constructed were also $100.0 \%$, but the PCCCC for 2,4-DNT was $99.67 \%$. In these cases, the false alarm rate was $0.5 \%$, and the sensitivity was $99.50 \%$. This result is an indication that samples of air or air with TATP were discriminated better than air with 2,4-DNT. In these cases, one of the air samples was poorly discriminated because this experiment was carried out in open path mode resulting in possible cross contamination from DNT in the laboratory. In this setup, interferences from ambient water vapor and $\mathrm{CO}_{2}$ were high. Therefore, in the model using the entire IR spectral region measured $\left(600-4000 \mathrm{~cm}^{-1}\right)$, it was necessary to eliminate the subspectral regions of $4000-3541,2384-2295$, and $1758-1490 \mathrm{~cm}^{-1}$, resulting in the improvement of the model, as summarized in Table 1.

In the third model (QCL scan), all of the samples were correctly classified. However, the number of samples analyzed must be considered, and the number of variables in this experiment is lower compared to the other experiments. The experimental conditions are not fully comparable because the intensity of this source is much higher than those of the other experiments and the sampling path is smaller for this system.

For the models to have highly significant statistical merit according to the canonical correlation coefficient $(P<$ 0.0001), the functions must have an excellent ability to determine the group differences. Wilks' lambda value indicates how many times the variance is not explained by group differences. Because these values were small, highly correlated differences were established.

Other models were generated using only the region of $873-1400 \mathrm{~cm}^{-1}$ or the region of emission of the QCL to compare the technique with the FT-IR-based experiments. The QCL-based experiments were better than the modulated source FT-IR setup, which, in turn, was better than the nonmodulated source FT-IR setup. The validation information is shown in Table 2. A high significance $(P<0.0001$, equivalent to $>99.99 \%$ confidence level) for all of the models was found, indicating that the resulting parameters are highly reliable and the comparison between techniques is highly dependable. The high power QCL scan produces a high sensitivity for trace level detection in air, but the modulated source FT-IR is close in performance to the QCL-based methodology. This result indicates that the modulation of light (using an interferometer) before reaching the sample increases the sensitivity [23] compared to a nonmodulated source FT-IR where the light first interacts with the sample prior to entering the interferometer. 
TABLE 2: Validation parameters for the models in the subspectral range of $850-1400 \mathrm{~cm}^{-1}$.

\begin{tabular}{|c|c|c|c|c|c|c|}
\hline \multirow{2}{*}{ Parameter } & \multicolumn{2}{|c|}{ Modulated FTIR (model 1) } & \multicolumn{2}{|c|}{ Unmodulated FTIR (model 2) } & \multicolumn{2}{|c|}{ QCL scan (model 3) } \\
\hline & TATP & DNT & TATP & DNT & TATP & DNT \\
\hline Wilks' lambda & 0.15 & 0.04 & 0.55 & 0.54 & 0.05 & 0.06 \\
\hline Canonical correlation & 0.92 & 0.98 & 0.67 & 0.65 & 0.98 & 0.97 \\
\hline PCCC & $99.74 \%$ & $99.98 \%$ & $92.00 \%$ & $93.33 \%$ & $100.00 \%$ & $100.00 \%$ \\
\hline PCCCC & $99.73 \%$ & $99.97 \%$ & $82.00 \%$ & $78.33 \%$ & $100.00 \%$ & $100.00 \%$ \\
\hline Sensibility & $100.00 \%$ & $100.00 \%$ & $81.00 \%$ & $77.00 \%$ & $100.00 \%$ & $100.00 \%$ \\
\hline Specificity & $93.33 \%$ & $99.87 \%$ & $84.00 \%$ & $81.00 \%$ & $100.00 \%$ & $100.00 \%$ \\
\hline False alarm & $0.00 \%$ & $0.00 \%$ & $16.00 \%$ & $19.00 \%$ & $0.00 \%$ & $0.00 \%$ \\
\hline Missed detection & $6.67 \%$ & $0.13 \%$ & $19.00 \%$ & $23.00 \%$ & $0.00 \%$ & $0.00 \%$ \\
\hline Latent variables (LV) & 10 & 10 & 10 & 10 & 5 & 5 \\
\hline$P$ value & $<0.0001$ & $<0.0001$ & $<0.0001$ & $<0.0001$ & $<0.0001$ & $<0.0001$ \\
\hline
\end{tabular}

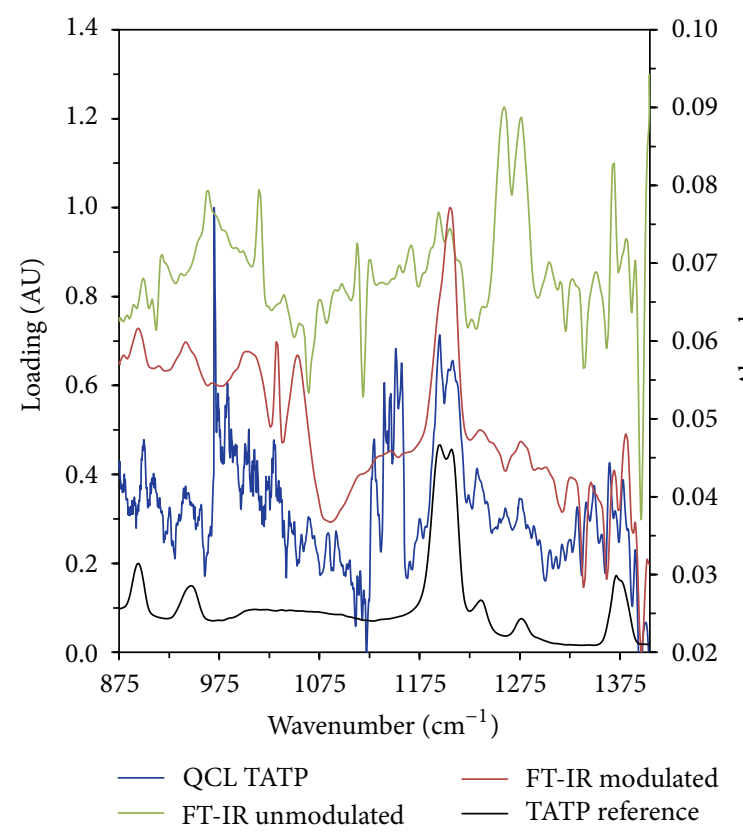

(a)

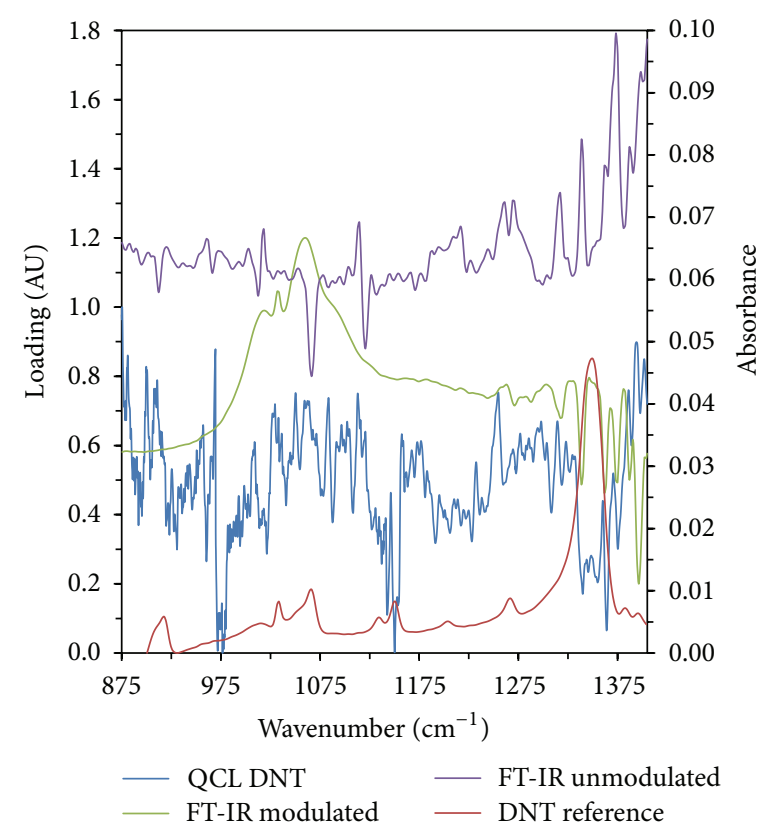

(b)

FIGURE 3: (a) First loadings for TATP models for the region $873-1400 \mathrm{~cm}^{-1}$. (b) First loadings for DNT models for the region $873-1400 \mathrm{~cm}^{-1}$. Reference gas-phase spectra included.

The loading for models of the region $\left(870-1400 \mathrm{~cm}^{-1}\right)$ are shown in Figure 3. The band observed for TATP at $1200 \mathrm{~cm}^{-1}$ that was tentatively assigned to $\mathrm{C}-\mathrm{O}$ stretching $[24,25]$ is the largest contributor to the loadings calculated by the models for modulated source FT-IR and QCL source. However, in the unmodulated source FT-IR experiments, the same result was not found: the first loading has the spectral information shifted. Other bands that contribute occur at $890 \mathrm{~cm}^{-1}$ and $939 \mathrm{~cm}^{-1}$, which are tentatively assigned to $\mathrm{O}-\mathrm{O}$ stretching $[24,25]$. The same analysis applies for all of the other loadings (data not shown). This indicates that the discrimination is generated by a combination of all of the loadings. The QCL model required only 5 latent variables (LV); FT-IR-based models required 7 (full spectrum) or 10 (spectral width) latent variables (LV).

4.1. Proof of the Presence of 2,4-DNT and TATP in Air at Trace Levels. The concentration of 2,4-DNT in air for different flow conditions and temperatures was calculated via a calibration curve obtained by GC- $\mu \mathrm{ECD}$ (Table 3 ). The presence of TATP was also established using GC-MS. To demonstrate the presence of the infrared signal in air, a small amount of TATP or 2,4-DNT $(0.1 \mu \mathrm{g})$ was deposited on a plate and introduced into a gas cell of $38.7 \mathrm{~cm}^{3}$ at a pressure of $0.0001 \mathrm{mBar}$. This procedure generated a density of $2538 \mathrm{pg} / \mathrm{mL}$ (in the worst case) when all of the explosive material sublimed and 
TABLE 3: Mass of 2,4-DNT determined by GC- $\mu \mathrm{ECD}$ for $2 \mathrm{~mL}$ of injected gas.

\begin{tabular}{lcccc}
\hline$T\left({ }^{\circ} \mathrm{C}\right)$ & $F(\mathrm{~mL} / \mathrm{s})$ & Peak area & Mass $(\mathrm{pg})$ & $\mathrm{pg} / \mathrm{mL}\left(\mu \mathrm{g} / \mathrm{m}^{3}\right)$ \\
\hline 20 & 1.6 & $7.564 . E+03$ & 98 & 49 \\
20 & 7.8 & $3.314 . E+03$ & 48 & 25 \\
20 & 15.7 & $3.078 . E+03$ & 46 & 23 \\
26 & 1.6 & $1.030 . E+05$ & 1202 & 601 \\
26 & 7.8 & $6.650 . E+04$ & 779 & 390 \\
26 & 15.7 & $5.720 . E+04$ & 672 & 336 \\
38 & 1.6 & $3.800 . E+05$ & 4407 & 2203 \\
15 & 15.7 & $2.793 . E+03$ & 42 & 21 \\
\hline
\end{tabular}

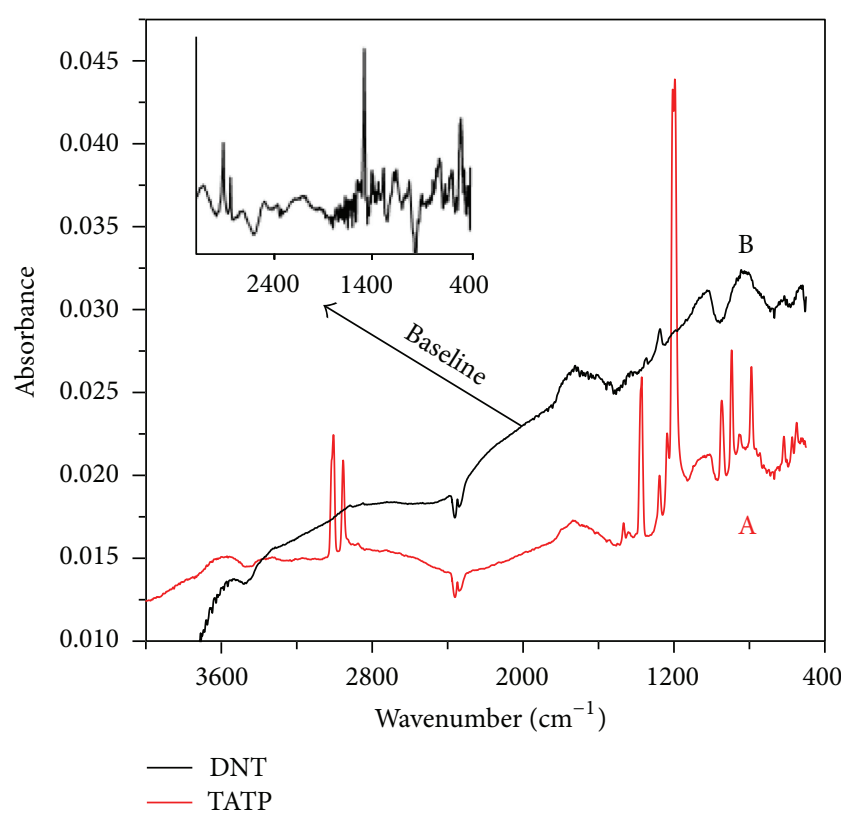

FIGURE 4: Low-pressure spectra in the gas phase with baseline correction of (A) TATP and (B) DNT.

negligible amounts were suctioned by the vacuum pump. This suggests that the concentration in the cell for the gas was $\ll 2538 \mathrm{pg} / \mathrm{mL}$. Spectra for the trace amounts of explosive were recorded to confirm that the detector was able to detect at this concentration level (Figure 4). This experiment was performed using the modulated source FT-IR system.

4.2. Limits of Detection. The low limits of detection (LOD) have been estimated for the two better performing techniques and their corresponding models: modulated source FT-IR and mid-IR (QC) laser source. In the case of the homemade explosive TATP, LOD values were estimated as $800 \mathrm{pg} / \mathrm{m}^{3}$ and $300 \mathrm{pg} / \mathrm{m}^{3}$ for modulated FT-IR and QCL-based detection, respectively. For the nitroaromatic military explosive 2,4DNT-LOD values were even lower: $31 \mathrm{pg} / \mathrm{m}^{3}$ and $0.7 \mathrm{pg} / \mathrm{m}^{3}$ for modulated source FT-IR and QCL source, respectively.

\section{Conclusions}

The results obtained in this research show that it is possible to determine the presence of peroxide-based explosives, such as TATP, and high sublimation pressure nitro compounds when they are in the gas phase mixed with air using PLSDA regression analysis of infrared spectral data. The QCLbased results exhibited a better capacity for discrimination or detection of the presence of explosives in air compared to other techniques. This result is due to the high power and collimation of the laser source increasing the sensitivity at trace level in air. It was also demonstrated that, when the light is modulated, an increase of sensitivity is obtained. Possible synergies between QCL sources, which are inherently modulated, and detection schemes could generate higher sensitivity techniques for gas phase sensing of hazardous chemicals. However, technical problems related to QCL scanning generated high noise levels. This resulted in unsuccessful efforts to modulate this source. A possible solution is to stop the QCL in a wavenumber range corresponding to emission bands within the target chemicals and then modulate the source and acquire the spectra at this position; next, move the QCL to another wavenumber central position close to the previous band and acquire the spectrum, and so on until the whole range of the QCL is scanned. This would provide the required sensitivity for the analysis at trace level.

\section{Acknowledgments}

Support from the US Department of Homeland Security under Award no. 2008-ST-061-ED0001 is acknowledged. However, the views and conclusions contained in this document are those of the authors and should not be interpreted as necessarily representing the official policies of the US Department of Homeland Security. This contribution was supported by the US Department of Defense, Prop. No. 58949-PH-REP, Agreement No. W911NF-11-1-0152. The authors also acknowledge contributions from Dr. Richard T. Hammond from Army Research Office, DoD. They gratefully acknowledge the contributions by Dr. Fred Haibach from Block Engineering in setting up the experimental part of the QCL-based experiments involving the LaserTune (Block Eng., Marlborough, MA, USA) and for his enlightening discussions.

\section{References}

[1] D. S. Moore, "Instrumentation for trace detection of high explosives," Review of Scientific Instruments, vol. 75, no. 8, pp. 2499-2512, 2004.

[2] D. S. Moore, "Recent advances in trace explosives detection instrumentation," Sensing and Imaging, vol. 8, no. 1, pp. 9-38, 2007.

[3] J. M. Sylvia, J. A. Janni, J. D. Klein, and K. M. Spencer, "Surfaceenhanced Raman detection of 2,4-dinitrotoluene impurity vapor as a marker to locate landmines," Analytical Chemistry, vol. 72, no. 23, pp. 5834-5840, 2000.

[4] K. J. Albert and D. R. Walt, "High-speed fluorescence detection of explosives-like vapors," Analytical Chemistry, vol. 72, no. 9, pp. 1947-1955, 2000. 
[5] E. R. Menzel, L. W. Menzel, and J. R. Schwierking, "A photoluminescence-based field method for detection of traces of explosives," The Scientific World Journal, vol. 4, pp. 725-735, 2004.

[6] Y. Salinas, R. Martínez-Máñez, M. D. Marcos et al., "Optical chemosensors and reagents to detect explosives," Chemical Society Reviews, vol. 41, no. 3, pp. 1261-1296, 2012.

[7] Y. Salinas, A. Agostini, É. Pérez-Esteve et al., "Fluorogenic detection of Tetryl and TNT explosives using nanoscopiccapped mesoporous hybrid materials," Journal of Materials Chemistry A, vol. 1, no. 11, pp. 3561-3564, 2013.

[8] G. K. Kannan, A. T. Nimal, U. Mittal, R. D. S. Yadava, and J. C. Kapoor, "Adsorption studies of carbowax coated surface acoustic wave (SAW) sensor for 2,4-dinitro toluene (DNT) vapour detection," Sensors and Actuators B, vol. 101, no. 3, pp. 328-334, 2004.

[9] R. Batlle, H. Carlsson, P. Tollbäck, A. Colmsjö, and C. Crescenzi, "Enhanced detection of nitroaromatic explosive vapors combining solid-phase extraction-air sampling, supercritical fluid extraction, and large-volume injection-GC," Analytical Chemistry, vol. 75, no. 13, pp. 3137-3144, 2003.

[10] C. Sánchez, H. Carlsson, A. Colmsjö, C. Crescenzi, and R. Batlle, "Determination of nitroaromatic compounds in air samples at femtogram level using C18 membrane sampling and on-line extraction with LC-MS," Analytical Chemistry, vol. 75, no. 17, pp. 4639-4645, 2003.

[11] R. Schulte-Ladbeck and U. Karst, "Determination of triacetonetriperoxide in ambient air," Analytica Chimica Acta, vol. 482, no. 2, pp. 183-188, 2003.

[12] J. I. Steinfeld and J. Wormhoudt, "Explosives detection: a challenge for physical chemistry," Annual Review of Physical Chemistry, vol. 49, no. 1, pp. 203-232, 1998.

[13] J. J. Perez, P. M. Flanigan, J. J. Brady, and R. J. Levis, "Classification of smokeless powders using laser electrospray mass spectrometry and offline multivariate statistical analysis," Analytical Chemistry, vol. 85, no. 1, pp. 296-302, 2013.

[14] F. C. de Lucia Jr. and J. L. Gottfried, "Influence of variable selection on partial least squares discriminant analysis models for explosive residue classification," Spectrochimica Acta B, vol. 66, no. 2, pp. 122-128, 2011.

[15] J. K. V. Mardia, J. T. Kent, and J. M. Biby, Chemometrics: Statistic and Computer Application in Analytical Chemistry, Academic Press, London, UK, 1980.

[16] K. R. Beebe, R. J. Pell, and M. B. Seasholtz, Chemometrics. A Pactricla Guide, John Wiley \& Sons, New York, NY, USA, 1998.

[17] C. J. Huberty, Applied Discriminant Analysis, WileyInterscience, Hoboken, NJ, USA, 1994.

[18] Y. M. Kim, J. F. MacGregor, and L. K. Kostanski, "Principal component analysis of FT-IR spectra for cationic photopolymerization of mixtures of two monomers," Chemometrics and Intelligent Laboratory Systems, vol. 75, no. 1, pp. 77-90, 2005.

[19] U. G. Indahl, N. S. Sahni, B. Kirkhus, and T. Næs, "Multivariate strategies for classification based on NIR-spectra-with application to mayonnaise," Chemometrics and Intelligent Laboratory Systems, vol. 49, no. 1, pp. 19-31, 1999.

[20] Y. Tan, L. Shi, W. Tong, G. T. G. Hwang, and C. Wang, "Multi-class tumor classification by discriminant partial least squares using microarray gene expression data and assessment of classification models," Computational Biology and Chemistry, vol. 28, no. 3, pp. 235-244, 2004.
[21] B. Lindholm-Sethson, S. Han, S. Ollmar et al., "Multivariate analysis of skin impedance data in long-term type 1 diabetic patients," Chemometrics and Intelligent Laboratory Systems, vol. 44, no. 1-2, pp. 381-394, 1998.

[22] Q. P. He, S. J. Qin, and J. Wang, "A new fault diagnosis method using fault directions in Fisher discriminant analysis," AIChE Journal, vol. 51, no. 2, pp. 555-571, 2005.

[23] L. C. Pacheco-Londoño, W. Ortiz-Rivera, O. M. PrimeraPedrozo, and S. P. Hernández-Rivera, "Vibrational spectroscopy standoff detection of explosives," Analytical and Bioanalytical Chemistry, vol. 395, no. 2, pp. 323-335, 2009.

[24] G. A. Buttigieg, A. K. Knight, S. Denson, C. Pommier, and M. B. Denton, "Characterization of the explosive triacetone triperoxide and detection by ion mobility spectrometry," Forensic Science International, vol. 135, no. 1, pp. 53-59, 2003.

[25] B. Brauer, F. Dubnikova, Y. Zeiri, R. Kosloff, and R. B. Gerber, "Vibrational spectroscopy of triacetone triperoxide (TATP): anharmonic fundamentals, overtones and combination bands," Spectrochimica Acta A, vol. 71, no. 4, pp. 1438-1445, 2008. 

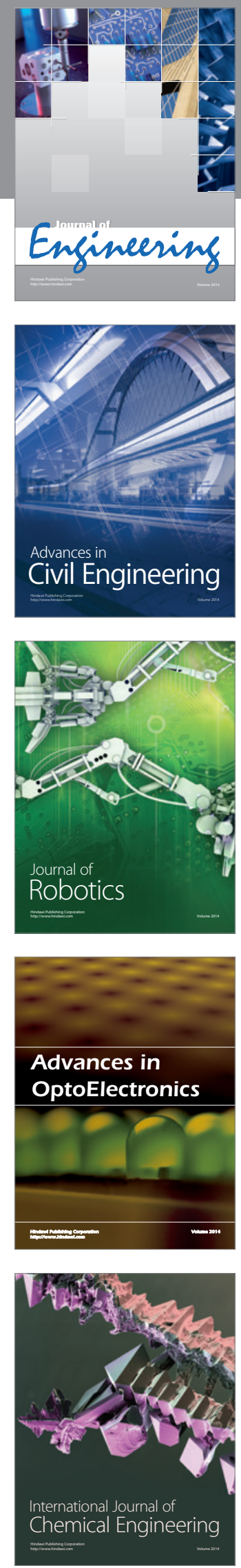

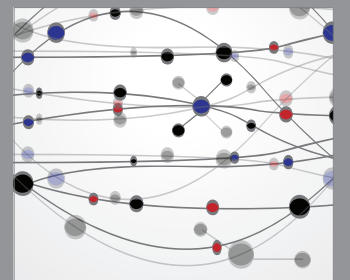

The Scientific World Journal
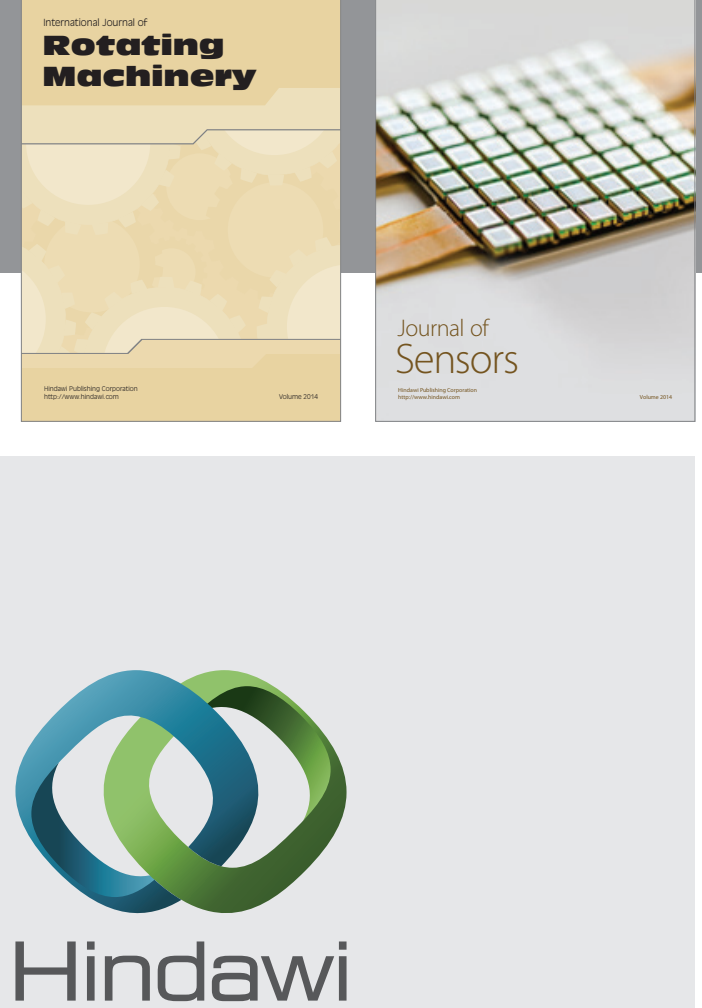

Submit your manuscripts at http://www.hindawi.com
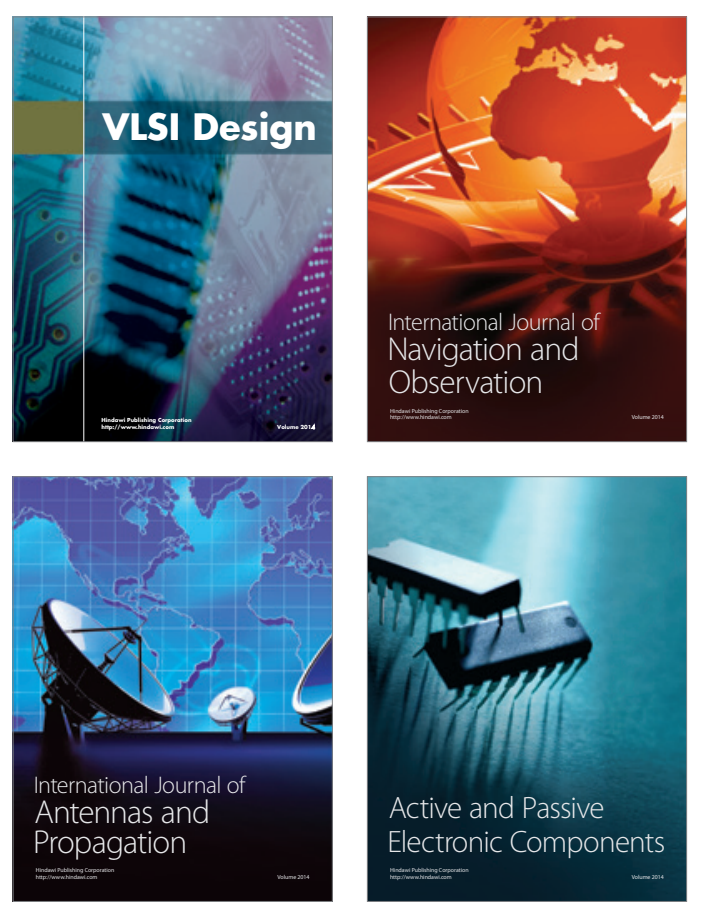
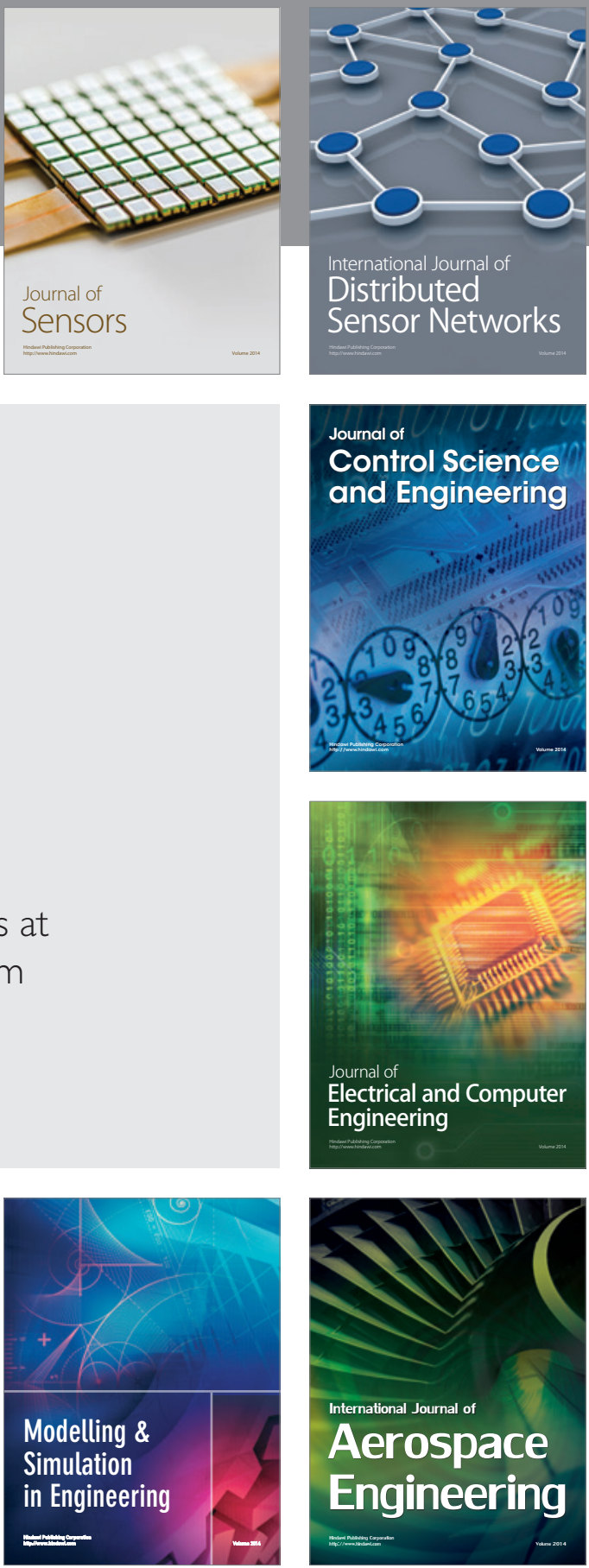

Journal of

Control Science

and Engineering
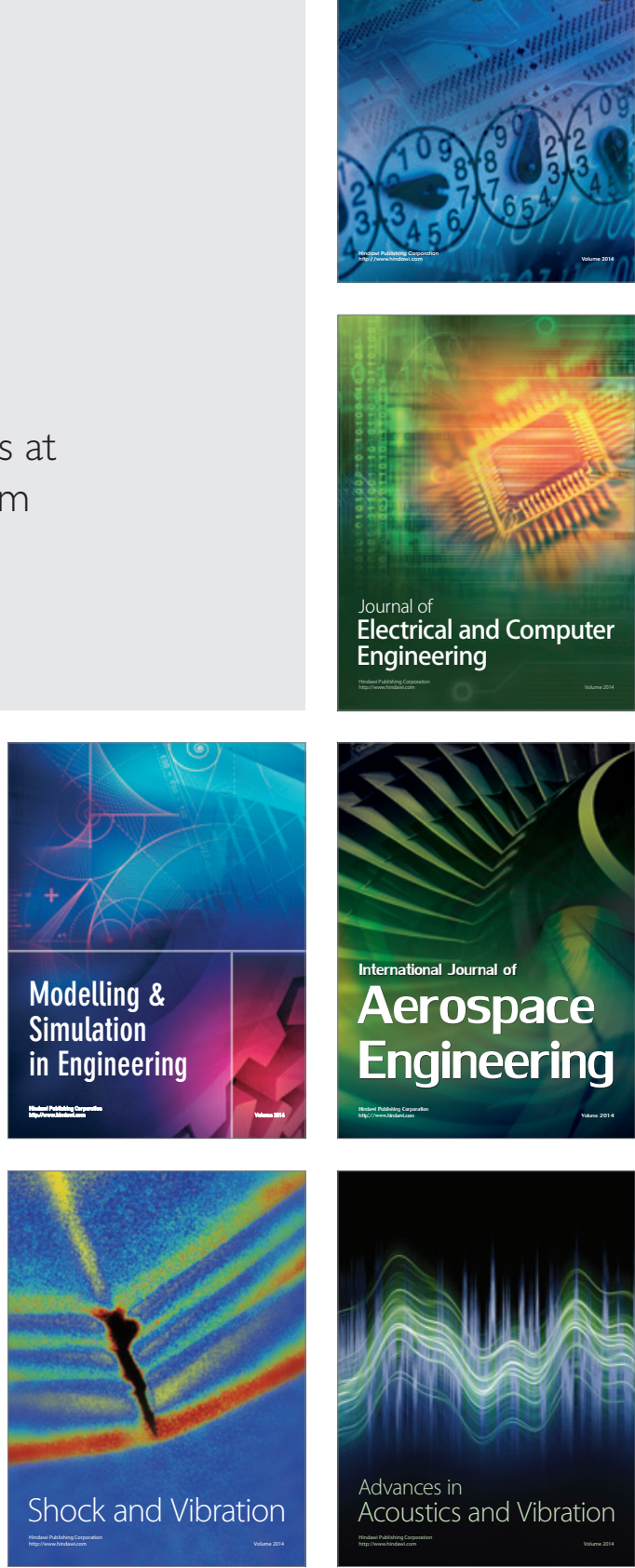